\title{
Subgroups of Finite Index in a Free Product With Amalgamated Subgroup
}

\author{
By W. W. Stothers
}

\begin{abstract}
Let $G$ be a free product of finitely many finite groups with amalgamated subgroup. Using coset diagrams, a recurrence relation is obtained for the number of subgroups, and of free subgroups, of each finite index in $G$. In the latter case, an asymptotic formula is derived. When the amalgamated subgroup is central, the relation takes a simpler form.
\end{abstract}

Introduction. Imrich [3] has given a formula for the number of subgroups of each finite index in $\operatorname{SL}(2, Z)$, the free product of $Z_{4}$ and $Z_{6}$ with amalgamated subgroup $Z_{2}$. Here we consider a free product of finite groups with an amalgamated subgroup. We obtain a recurrence relation similar to that in [3], though, in the general case, we cannot derive an asymptotic formula. More complete results are obtained for the number of free subgroups of such a group. Our methods would extend easily to a "graph of groups", but the notation would become (more) unpleasant. In the case of $\operatorname{SL}(2, Z)$, the amalgamated subgroup is central and a simpler treatment is possible; we hope to publish the details elsewhere.

1. Coset Diagrams. Suppose that $M$ is a group with presentation $\langle m \in \mathfrak{N}$ : $r=1, r \in \Re>$. We may as well assume that $m^{-1} \in \mathfrak{N}$, whenever $m \in \mathfrak{N}$.

Let $K<M$. The coset diagram $\mathscr{D}=\mathscr{D}(K)$ for $K$ is a directed pseudograph with vertices labelled by the cosets $K m$ and edges labelled by the elements of $\mathfrak{N}$. For $m \in \mathfrak{N}$, the $m$-edges indicate the effect of post-multiplication by $m$ on the cosets. Write $V(\mathscr{D})$ for the set of vertices of $\mathscr{D}$. Then, by the definition of edges:

(A) for $m \in \mathfrak{N}, P \in V(\mathscr{D})$, there is one $m$-edge into $P$ and one out of $P$.

If $w=m_{1} \cdots m_{s}$ is a word over $\mathfrak{N}$ and $P \in V(\mathscr{D})$, then there is a walk $w(P)$ from $P$ defined in the obvious way (follow the $m_{1}$-edge, $e$, out of $P$, the $m_{2}$-edge from the end of $e$, and so on). With this notation, we also have

(B) for $r \in R, P \in V(\mathscr{D})$, the walk $r(P)$ ends at $P$.

If words $w_{1}, w_{2}$ over $\mathfrak{T}$ give the same element of $M$, then (B) implies that, for $P \in V(\mathscr{D}), w_{1}(P)$ and $w_{2}(P)$ end at the same vertex. Hence, $m \in M$ gives a permutation $\pi_{m}$ of $V(\mathscr{D}), \pi_{m}(P)$ being the end of $w(P)$ where $w$ is any word representing $m$. If $P_{0}$ is the vertex labelled by the coset $K 1$, then

$$
K=\left\{m \in M: \pi_{m}\left(P_{0}\right)=P_{0}\right\}, \quad K n=\left\{m \in M: \pi_{m}\left(P_{0}\right)=\pi_{n}\left(P_{0}\right)\right\} .
$$

Suppose that $m \in M$ and that the word $w$ represents $m$. Then $w\left(P_{0}\right)$ is a walk from $P_{0}$ to the vertex labelled by $K m$. Hence,

(C) $\mathscr{D}(K)$ is connected.

Received July 25, 1980.

AMS (MOS) subject classifications (1970). Primary 20E30; Secondary 20H10, $10 \mathrm{D} 05$. 
Definition. An $M$-diagram is a directed pseudograph, edge-labelled by $\mathfrak{N}$ which is such that (A) and (B) hold.

Note that, from (A), (B), each element of $M$ defines a permutation of the vertex set of an $M$-diagram.

Definition. Suppose that $\mathscr{D}$ is a connected $M$-diagram and that $P \in V(\mathscr{D})$. Then,

$$
[\mathscr{Q}, P]=\left\{m \in M: \pi_{m}(P)=P\right\} \text {. }
$$

It is clear that $[\mathscr{D}, P]$ is a subgroup of $M$. Labelling the vertices by the cosets, it is clear that $\mathscr{D}=\mathscr{D}([\mathscr{D}, P])$ and $|V(\mathscr{D})|=|[\mathscr{D}, P]: M|$. If $Q \in V(\mathscr{D})$, then, as $\mathscr{D}$ is connected, there is an $m \in M$ with $\pi_{m}(P)=Q$. Clearly, $[\mathscr{D}, Q]=[\mathscr{D}, P]^{m}$, and each conjugate arises in this way, so that

Proposition 1.1. For a group $M$, there is a one-to-one correspondence between connected $M$-diagrams with $m$ vertices and conjugacy classes of subgroups of index $m$ in $\boldsymbol{M}$.

If $\mathscr{D}$ is a connected $M$-diagram and $P, Q \in V(\mathscr{D})$, then it may happen that $[\mathscr{Q}, P]=[\mathscr{Q}, Q]$. The number of $Q$ with this property is equal to the index of $[\mathscr{D}, P]$ in its normalizer. Since this is not affected by conjugation, the number is independent of the choice of $P$.

Definitions. (1) For a connected $M$-diagram $\mathscr{D}, c(\mathscr{D})$ is the number of vertices which give the same subgroup.

(2) An $M$-diagram of order $n$ is an $M$-diagram with $n$ vertices labelled by $\{1, \ldots, n\}$.

Suppose that $\mathscr{D}$ is a connected $M$-diagram of order $n$. The vertices of $\mathscr{D}$ are implicitly labelled by the cosets of $[\mathscr{D}, 1]$. Thus, a permutation of the labels $\{2, \ldots, n\}$ must give a different $M$-diagram of order $n$. A permutation $\sigma$ of $\{1, \ldots, n\}$ gives the same diagram of order $n$ if and only if

(i) $[\mathscr{Q}, \sigma(1)]=[\mathscr{D}, 1]$, and

(ii) $\left.\sigma\right|_{\{2, \ldots, n\}}$ is the permutation induced by the coset labellings for $[\mathscr{D}, 1]$ and for $[\mathscr{Q}, \sigma(1)]$.

It follows that exactly $c(\mathscr{D})$ permutations give the same diagram of order $n$. We have proved

Proposition 1.2. (1) A subgroup of index $n$ in $M$ corresponds to $(n-1)$ ! $M$-diagrams of order $n$.

(2) A connected $M$-diagram $\mathscr{D}$ of order $n$ can be labelled, using $\{1, \ldots, n\}$, in $n ! / c(\circlearrowleft)$ different ways.

Definition. A property $\mathscr{P}$ of pseudographs is heritable if whenever $\mathscr{D}$ has property $\mathscr{P}$ so has each union of components of $\mathscr{D}$.

In an $M$-diagram, each relator gives a closed walk (by (B)), so this lies in a single component. Hence, the property, $\mathscr{P}(M)$, of being an $M$-diagram is heritable.

Definition. Suppose that $\mathcal{P}$ is a heritable property. Then $E(\mathscr{P}, n)$ is the number of pseudographs with property $\mathcal{P}$ with vertices labelled by $\{1, \ldots, n\} . E_{0}(\mathscr{P}, n)$ is the number which is connected. 
We note that these numbers will be finite, provided that $\mathscr{P}$ implies that the number of edges at each vertex is bounded by a constant $K(\mathscr{P})$. This will be true of $\mathscr{P}(M)$, provided that there is a finite generating set $\mathfrak{T}$.

Considering the component containing the vertex 1 in each diagram (as in [6]), it is easy to prove

Proposition 1.3. Let $\mathcal{P}$ be a heritable property such that $E(\mathscr{P}, n)$ is finite for each n. Then

$$
E_{0}(\mathscr{P}, n)=E(\mathscr{P}, n)-\sum_{k=1}^{n-1}\left(\begin{array}{l}
n-1 \\
k-1
\end{array}\right) E_{0}(\mathscr{P}, k) E(\mathscr{P}, n-k)
$$

Definition. For a group $M, N(M, n)$ is the number of subgroups of index $n$ in $M$.

Provided that $M$ is finitely generated, each $N(M, n)$ is finite. Applying Proposition 1.2(1),

$$
N(M, n)=E_{0}(\mathscr{P}(M), n) /(n-1) !
$$

Combining this with Proposition 1.3, we have

THEOREM 1.4. For a finitely generated group $M$,

$$
N(M, n)=n(E(\mathscr{P}(M), n) / n !)-\sum_{k=1}^{n-1} N(M, k) E(\mathscr{P}(M), n-k) /(n-k) ! .
$$

After this, we concentrate on evaluating the $E(\mathscr{P}, n)$.

2. Free Products With Amalgamated Subgroup. We consider the group $G$, defined by

$$
G={ }^{*} c\{G(i): i=1, \ldots, s\}
$$

where $s>2$ and, for each $i, G(i)$ is a finite group. To avoid trivial cases, we assume that, for each $i,|G(i) / C|>2$. We take the finite set of generators $\mathcal{G}=\cup G(i)$. Then $g^{-1} \in \mathcal{G}$ when $g \in \mathcal{G}$, and there is a set of relators each of which involves symbols from a single $G(i)$.

Definition. Let $\mathscr{D}$ be a $G$-diagram. Then $\mathscr{D}_{0}($ resp. $\mathscr{D}(i), 1<i<s)$ is the subpseudograph with vertex set $V(ゆ)$ and edge set consisting of the $g$-edges for $g \in C$ (resp. $g \in G(i)$ ).

It is clear that $\mathscr{D}_{0}$ (resp. $\left.\mathscr{D}(i)\right)$ is a $C$ - (resp. $\left.G(i)-\right)$ diagram. In a similar way, we obtain a $C$-diagram $\mathcal{E}_{0}$ from a $G(i)$-diagram $\mathcal{E}$.

In a case where $C=\{1\}$, i.e. $G$ is a free product, a $G$-diagram can be obtained from a collection $\mathscr{D}(1), \ldots, \mathscr{D}(s)$, where each $\mathscr{D}(i)$ is a $G(i)$-diagram with a fixed vertex set $\delta$. In general, we note that, if $\mathscr{D}$ is a $G$-diagram, then, for $i=1, \ldots, s$, $\mathscr{D}_{0}=\mathscr{D}(i)_{0}$, so that all the $\mathscr{D}(i)_{0}$ are identical. Conversely, given a collection of $G(i)$-diagrams, with a common vertex and identical induced $C$-diagrams, we get a $G$-diagram. We have proved

Proposition 2.1. Suppose that $\mathcal{S}$ is a finite set and that, for $i=1, \ldots, s, \mathscr{D}_{i}$ is a $G(i)$-diagram with $V\left(\mathscr{D}_{i}\right)=\mathcal{S}$. Then there is a G-diagram $\mathscr{D}$ with $\mathscr{D}(i)=\mathscr{D}_{i}$ if and only if, for $1<i, j<s,\left(\mathscr{D}_{i}\right)_{0}=\left(\mathscr{D}_{j}\right)_{0}$. Further, if $\left\{\mathscr{D}_{i}\right\}$ and $\left\{\mathcal{E}_{i}\right\}$ yield $G$-diagrams $\mathscr{D}, \mathcal{E}$, then $\mathscr{D}=\mathcal{E}$ if and only if, for each $i, \mathscr{D}_{i}=\mathcal{E}_{i}$. 
This allows us to compute the $E(\mathscr{P}(G), n)$ from the $E(\mathscr{P}(G(i)), n)$.

We note that the $G(i)$, and hence $C$, are finite groups. From Proposition 1.2(1), each has a finite collection of connected diagrams.

Definitions. (1) Let $\mathcal{C}(1), \ldots, \mathcal{C}(m(0))$ be the set of connected $C$-diagrams, with $\mathcal{C}(1)$ corresponding to $\{\{1\}\}$. Let $c(k)=c(\mathcal{C}(k)), g(k)=|V(\mathcal{C}(k))|$. (Then $g(k)$ is the index of any representative subgroup, and $g(k) / c(k)$ is the size of the conjugacy class. Also, $c(1)=g(1)=|C|$.)

(2) For $i=1, \ldots, s$, let $\mathcal{G}(i, 1), \ldots, \mathcal{G}(i, m(i))$ be the set of connected $G(i)$ diagrams, with $\mathcal{G}(i, 1)$ corresponding to $\{\{1\}\}$. Let $c(i, j)=c(\mathcal{G}(i, j))$ and $g(i, j)=$ $|V(\mathcal{G}(i, j))|$. (For each $i, c(i, 1)=g(i, 1)=|G(i)|$.)

Any $M$-diagram consists of a number of components. As noted above, each component is a connected $M$-diagram.

Definition. Suppose that $\mathcal{C}$ is a $C$-diagram consisting of $r[\mathcal{C}](k)$ copies of $\mathcal{C}(k)$, $k=1, \ldots, m(0)$. Then $\mathcal{C}$ is of $C$-type $r[\mathcal{C}]=(r[\mathcal{C}](1), \ldots, r[\mathcal{C}](m(0)))$.

As $\mathcal{C}(k)$ has $g(k)$ vertices, a diagram of $C$-type $\mathbf{r}$ has $n$ vertices, where

$$
n=\sum_{k} r(k) g(k) \text {. }
$$

Since a $G$ - (or $G(i)$-) diagram defines a $C$-diagram, we can talk of the $C$-type of such a diagram.

Definition. For relevant $i, j, k, s(i, j, k)$ is the number of copies of $C(k)$ in $\mathcal{G}(i, j)_{0}$.

Definition. Let $i \in\{1, \ldots, s\}$. Suppose that $\mathscr{D}$ is a $G(i)$-diagram with $w[\mathscr{D}](i, j)$ copies of $\mathcal{G}(i, j)$. Then $\mathscr{D}$ is of $i$-type $w[\mathscr{D}](i)=(w[\mathscr{D}](i, 1), \ldots, w[\mathscr{D}](i, m(i)))$.

A $G(i)$-diagram of $i$-type $w(i)$ has $C$-type $\mathbf{r}$, where, for each $k$,

$$
r(k)=\sum_{j} w(i, j) s(i, j, k) \text {. }
$$

Definitions. (1) For each $n, S(n)$ is the set of $\mathbf{r}$ such that (2) holds.

(2) For $\mathbf{r} \in S(n), T(i, \mathbf{r})$ is the set of $\mathbf{w}(i)$ such that (3) holds for all $k$.

Then $S(n)$ consists of the $C$-types which are possible for diagrams with $n$ vertices, and, for each $i, T(i, \mathbf{r})$ consists of the $i$-types which are compatible with the $C$-type $\mathbf{r}$.

Suppose that $\mathscr{D}$ is a $G$-diagram of $C$-type $\mathbf{r}$ and $i$-type $w(i), i=1, \ldots, s$. Let $\boldsymbol{n}=|V(\mathscr{D})|$. We consider the labelling of $\mathscr{D}$ by $\{1, \ldots, n\}$. We can split $\{1, \ldots, n\}$ into $r(k)$ subsets of size $g(k), k=1, \ldots, m(0)$, in

$$
n ! / \prod_{k}\left\{r(k) !(g(k) !)^{r(k)}\right\}
$$

different ways. By Proposition 1.2(2), each $\mathcal{C}(k)$ can be labelled $g(k) ! / c(k)$ ways by a fixed set of size $g(k)$. Hence $\mathscr{D}_{0}$ can be labelled in

$$
L_{0}(\mathbf{r})=n ! / \prod_{k}\left\{r(k) ! c(k)^{r(k)}\right\}
$$

ways. For $i \in\{1, \ldots, s\}$, we can argue as for (4) to see that $\mathscr{D}(i)$ can be labelled in

$$
L_{i}(\mathbf{w}(i))=n ! / \prod_{j}\left\{w(i, j) ! c(i, j)^{w(i, j)}\right\}
$$


ways. We have considered all labellings of $\mathscr{D}(i)$, so each induced labelling of $\mathscr{D}_{0}$ occurs equally of ten, so that we have

Proposition 2.2. Suppose that $\mathrm{r} \in S(n)$ and that $\mathrm{w}(i) \in T(i, \mathrm{r})$. Let $\mathcal{E}$ be a $C$-diagram of order $n$ and $C$-type $\mathbf{r}$. Then the number of $G(i)$-diagrams $\mathscr{D}$, of order $n$ and $i$-type $w(i)$ such that $\mathscr{D}_{0}=\mathcal{E}$, is $L_{i}(\mathbf{w}(i)) / L_{0}(\mathbf{r})$.

Combining Propositions 2.1 and 2.2, we get

THEOREM 2.3. Suppose that $G$ is defined by (1). Then the number of G-diagrams of order $n$ is

$$
n ! \sum_{\mathbf{r} \in S(n)}\left\{r(k) ! c(k)^{r(k)}\right\}^{s-1} \prod_{i=1}^{s} \sum_{T(i, \mathbf{r})}\left\{w(i, j) ! c(i, j)^{w(i, j)}\right\}^{-1} .
$$

Proof. By definition, $S(n)$ includes all possible $C$-types for diagrams of order $n$. For each $\mathbf{r} \in S(n), T(i, \mathbf{r})$ includes all $\mathbf{w}(i)$ which are compatible with $\mathbf{r}$. A $C$-diagram of $C$-type $\mathbf{r}$ can be labelled in $L_{0}(\mathbf{r})$ ways; see (4). For each labelling, there are $L_{i}(\mathbf{w}(i)) / L_{0}(\mathbf{r})$ ways of making this into a $G(i)$-diagram of $i$-type $w(i)$. Hence the number of $G$-diagrams of order $n$, which are of $C$-type $\mathbf{r}$ and $i$-type w( $(i)$, $i=1, \ldots, s$, is

$$
L_{0}(\mathbf{r}) \cdot \prod_{i=1}^{s} L_{i}(\mathbf{w}(i)) / L_{0}(\mathbf{r})=\left(L_{0}(\mathbf{r})\right)^{1-s} \prod_{i=1}^{s} L_{i}(\mathbf{w}(i)) .
$$

The result follows from (4) and (5).

The number obtained in Theorem 2.3 is, of course $E(\mathscr{P}(G), n)$. Using Theorems 2.3 and 1.4, we get a recurrence relation for $N(G, n)$ involving the $E(\mathscr{P}(G), m) / m$ ! for $m<n$. This can be used to evaluate the $N(G, n)$ successively. To indicate the type of result, we give an example.

Example. The extended modular group $\bar{\Gamma}=\operatorname{PGL}(2, Z)$ has the presentation (see pp. 85-86 of [1])

$$
\bar{\Gamma}=\left\langle t, u, v: t^{2}=u^{2}=v^{3}=(t u)^{2}=(t v)^{3}=1\right\rangle .
$$

If we put $a=t u, b=t v$, then

$$
\begin{gathered}
\bar{\Gamma}=\left\langle a, b, t: a^{2}=b^{3}=t^{2}=(a t)^{2}=(b t)^{2}=1\right\rangle, \\
\bar{\Gamma} \simeq G(1){ }^{*} C G(2),
\end{gathered}
$$

where $G(1)=\langle a, t\rangle$, the Klein 4-group, and $G(2)=\langle b, t\rangle$, the symmetric group on three symbols, and $C=\langle t\rangle \simeq Z_{2}$. We have

$$
\begin{array}{ll}
\mathcal{C}(1)=\{\{1\}\}, & c(1)=g(1)=2, \\
\mathcal{C}(2)=\{C\}, & c(2)=g(2)=1 .
\end{array}
$$

For each $G(i)$, we list the pertinent facts in the form

$$
(G(i, j), c(i, j), g(i, j), s(i, j, 1), s(i, j, 2)),
$$

where $G(i, j)$ is a group in the class corresponding to $\mathcal{G}(i, j)$. In this case, $m(0)=2$, and the last two entries give the $C$-type of $\mathcal{G}(i, j)$. 
For $i=1, m(1)=5$ and we have the data

$$
\begin{aligned}
& (\{1\}, 4,4,2,0), \\
& (\langle a\rangle, 2,2,1,0), \\
& (\langle t\rangle, 2,2,0,2), \\
& (\langle a t\rangle, 2,2,1,0), \\
& (G(1), 1,1,0,1) .
\end{aligned}
$$

For $i=2, m(2)=4$ and we have the data

$$
\begin{aligned}
& (\{1\}, 6,6,3,0), \\
& (\langle t\rangle, 1,3,1,1), \\
& (\langle b\rangle, 2,2,1,0), \\
& (G(2), 1,1,0,1) .
\end{aligned}
$$

Then, from the definitions,

$$
\begin{gathered}
S(n)=\left\{\left(r_{1}, r_{2}\right): 2 r_{1}+r_{2}=n\right\}, \\
T\left(1,\left(r_{1}, r_{2}\right)\right)=\left\{\left(x_{1}, \ldots, x_{5}\right): 2 x_{1}+x_{2}+x_{4}=r_{1}, 2 x_{3}+x_{5}=r_{2}\right\}, \\
T\left(2,\left(r_{1}, r_{2}\right)\right)=\left\{\left(y_{1}, \ldots, y_{4}\right): 3 y_{1}+y_{2}+y_{4}=r_{1}, y_{2}+y_{4}=r_{2}\right\} .
\end{gathered}
$$

Then $E(\mathscr{P}(\bar{\Gamma}), n)$ is given by an eleven-fold sum. The conditions on the elements of $S(n)$ and the $T(i, \mathbf{r})$ enable us to reduce this to a six-fold sum, though the presence of $y_{2}$ in both sums for $T(2, \mathbf{r})$ seems to preclude an elegant formula. We omit the actual result but observe that, even in this comparatively simple case, the formula is complex. It is doubtful if a good estimate can be made.

3. Central Amalgamated Subgroup. The calculations are rather simpler when $C$ is central in $G$.

LEMMA 3.1. Suppose that $C$ is a finite central subgroup of a group G. For a connected G-diagram, all components of the induced $C$-diagram are identical.

Proof. Suppose that $\mathscr{D}$ is a connected $G$-diagram and that $\mathscr{D}_{0}$ is the induced $C$-diagram (we may as well assume that $C$ is a subset of the chosen generating set for $G$ ).

If $\mathscr{D}_{0}$ is connected, there is nothing to prove.

Suppose that $\mathcal{E}_{1}$ and $\mathcal{E}_{2}$ are components of $\mathscr{D}_{0}$. We choose vertices $P$ on $\mathcal{E}_{1}$ and $Q$ on $\mathcal{E}_{2}$. Then $C_{1}=\left[\mathcal{E}_{1}, P\right]$ and $C_{2}=\left[\mathcal{E}_{2}, Q\right]$ are subgroups of $C$. As $\mathcal{E}_{1} \subseteq \mathscr{D}$, $C_{1} \subseteq[\mathscr{D}, P]$. If $g \in C \cap[\mathscr{D}, P]$, then, as $g \in C$, it is contained in the generating set, and we may take a corresponding path consisting of a single edge (the $g$-edge out of $P$ ). As $\varepsilon_{1}$ is a $C$-diagram, this edge belongs to $\varepsilon_{1}$. As $g \in[\mathscr{D}, P]$, any path for $g$ is closed, so that $g \in\left[\mathcal{E}_{1}, P\right]=C_{1}$. Hence, $C_{1}=C \cap[\mathscr{Q}, P]$. Similarly, $C_{2}=C \cap[\mathscr{D}, Q]$. Since $\mathscr{D}$ is connected, there is an element $q \in G$ such that $\pi_{q}(Q)=P$. Then $[\mathscr{D}, P]=[\mathscr{D}, Q]^{q}$, so that $C_{2}^{q}=C^{q} \cap[\mathscr{D}, P]$. As $C$ is central, $C^{q}=C$, and hence $C_{2}^{q}=C_{2}$. Thus, $C_{2}=C \cap[\mathscr{D}, P]=C_{1}$. Since these are subgroups defined by $\varepsilon_{1}$ and $\varepsilon_{2}$, we must have $\varepsilon_{1}=\delta_{2}$, and the lemma is proved.

From now on, we shall assume that $G$ is defined by (1) and that $C$ is central in $G$ (and so also in each $G(i)$ ). Then, as $C$ is abelian, each $C(k)$ corresponds to a single (normal) subgroup, $C(k)$ say, and $c(k)=g(k)=|C: C(k)|$. From the proof of Lemma 3.1, the diagram $\mathscr{D}_{0}$ consists of copies of $C(k)$, where $k$ is such that 
$C(k)=C \cap[\mathscr{D}, P]$. Thus, the $C$-type of a connected $G$-diagram has exactly one nonzero entry.

Definitions. (1) For $k \in\{1, \ldots, m(0)\}$, let $\mathscr{P}_{k}(G)$ be the property of being a $G$-diagram with $C$-type $\mathrm{r}$ such that $r(i)=0, i \neq k$.

It is clear that $\mathscr{P}_{k}(G)$ is heritable.

(2) For $k \in\{1, \ldots, m(0)\}$, let $N_{k}(G, n)$ be the number of subgroups $H$ of index $n$ in $G$ such that $C \cap H=C(k)$.

From Proposition 1.2(1), we obtain

$$
N_{k}(G, n)=E_{0}\left(\mathscr{P}_{k}(G), n\right) /(n-1) !
$$

and hence an analogue of Proposition 1.3. The $E\left(\mathscr{P}_{k}(G), n\right)$ are easier to compute than the $E(\mathcal{P}(G), n)$ since the relevant $i$-types have $w(i, j) \neq 0$ only if $\mathcal{G}(i, j)_{0}$ consists of copies of $\mathcal{C}(k)$. Thus, we can split the $\mathcal{G}(i, j)$ into those relevant to each $k$. The evaluation of the $E\left(\mathscr{P}_{k}(G), n\right)$ involve those of just one class.

Example. Let

$$
G=\left\langle a_{1}, a_{2}: a_{1}^{4}=a_{2}^{4}=a_{1}^{2} a_{2}^{2}=1\right\rangle .
$$

Then $G$ is isomorphic to the free product of two copies of $Z_{4}$ (e.g. $\left\langle a_{1}\right\rangle$ and $\left\langle a_{2}\right\rangle$ ) with amalgamated subgroup $Z_{2}\left(\left\langle a_{1}^{2}\right\rangle=\left\langle a_{2}^{2}\right\rangle\right)$. In this example, all groups involved are abelian, so each conjugacy class consists of a single subgroup. We have $m(0)=2$ and

$$
\begin{array}{ll}
C(1)=\{1\}, & c(1)=g(1)=2, \\
C(2)=\left\langle a_{2}^{2}\right\rangle, & c(2)=g(2)=1 .
\end{array}
$$

With the notation of the previous example, we have, for $i=1,2, m(i)=3$ and the data

$$
\begin{aligned}
& (\{1\}, 4,4,2,0), \\
& \left(\left\langle a_{i}^{2}\right\rangle, 2,2,0,2\right), \\
& \left(\left\langle a_{i}\right\rangle, 1,1,0,1\right) .
\end{aligned}
$$

Subgroups $H$ with $H \cap C=\{1\}$ involve only $C(1)$ and hence only the $G(i, 1)$, so that $E\left(\mathscr{P}_{1}(G), n\right)$ is zero unless $n$ is divisible by 4 . The argument for Theorem 2.3 yields

$$
E\left(\mathscr{P}_{1}(G), 4 m\right)=(4 m) !(2 m) ! /\left(2^{m} m !\right)^{2} .
$$

It follows that $N_{1}(G, n)$ is zero unless 4 divides $n$, and

$$
\begin{aligned}
N_{1}(G, 4 m)= & 4 m(2 m) !\left(2^{m} m !\right)^{-2} \\
& -\sum_{k=1}^{m-1} N_{1}(G, 4 k)(2(n-k)) !\left(2^{n-k}(n-k) !\right)^{-2} .
\end{aligned}
$$

For $m=1$, the sum is empty, so that $N_{1}(G, 4)=2$. Assume (inductively) that $N_{1}(G, 4 i)=2, i=1, \ldots, m-1$. Then the sum in (8) becomes

$$
2 \sum_{k=1}^{m-1}(2 k) !\left(2^{k} k !\right)^{-2}
$$


From Section 2 of [5], we have the identity (omitting the erroneous "!" on the right)

$$
(2 m+1)\left(2^{m} m !\right)^{-2}=\sum_{j=0}^{m}(2 j) !\left(2^{j} j !\right)^{-2} .
$$

Then (8) yields $N_{1}(G, 4 m)=2$. Hence $N_{1}(G, 4 m)=2$ for all $m$.

For $N_{2}(G, n)$, we could proceed using the $E\left(\mathscr{P}_{2}(G), n\right)$. Now only $G(i, 2)$ and $G(i, 3)$ can occur, and the induced $C$-diagrams are loops. We obtain

$$
E\left(\mathscr{P}_{2}(G), n\right)=n !\left\{\sum_{k=0}^{[n / 2]}\left(k !(n-2 k) ! 2^{k}\right)^{-1}\right\}^{2} \text {. }
$$

However, it is quicker to observe that, in the notation of [4], $E\left(\mathscr{P}_{2}(G), n\right)=$ $\left(\tau_{2}(n)\right)^{2}$. By the results of that paper, $N_{2}(G, n)$ is equal to the number of subgroups of index 2 in $Z_{2} * Z_{2}$ (both satisfy the same recurrence). The latter number is given in [5], and it follows that

$$
N_{2}(G, n)= \begin{cases}n, & n \text { odd, } \\ n+1, & n \text { even }\end{cases}
$$

As a footnote, we observe that the relationship between $N_{2}(G, n)$ and $N\left(Z_{2} * Z_{2}, n\right)$ is not accidental. Noting that $G / C \simeq Z_{2} * Z_{2}$ in this case, the reader should be able to find a short (algebraic) proof of the relationship we 'discovered' in the calculation.

4. Free Subgroups. As we might expect on comparing [4] with [5], we can get more detailed results for the number of free subgroups.

Definition. For a group $G$, let $N_{0}(G, n)$ be the number of free subgroups of index $n$ in $G$.

Throughout this section, we shall assume that $G$ is defined by (1). We shall evaluate $N_{0}$ by means of a suitable heritable property.

LEMMA 4.1. A G-diagram $\mathscr{D}$ corresponds to a class of free subgroups if and only if, for $i=1, \ldots, s, \mathscr{D}(i)$ consists of copies of $\mathcal{G}(i, 1)$ (the diagram for the trivial subgroup).

Proof. We observe that, as $C$ is finite, a free subgroup $H$ cannot have nontrivial intersections with $C$ or with any of its $G$-conjugates, i.e. for each $g \in G$,

$$
H \cap C^{8}=\{1\} \text {. }
$$

Then the theorem of H. Neumann, quoted in [2], applies to show that the subgroup $H$ (since it satisfies (9)) is the free product of a free group and conjugates of subgroups of the $G(i)$. Thus, if $H$ is free, these subgroups must be trivial, since the $G(i)$ are finite. Conversely, if $H$ has trivial intersection with each conjugate of each $G(i)$, then (9) holds (as $C \subseteq G(1)$ ). Then, again by Neumann's theorem, $H$ is free. From the proof of the theorem given in [2], the condition we have obtained is equivalent to that in the statement of the lemma.

Definition. Let $\mathscr{P}_{0}(G)$ be the property that a pseudograph $\mathscr{D}$ is a $G$-diagram such that, for each $i, \mathscr{D}(i)$ consists of copies of $\mathcal{G}(i, 1)$.

Clearly, $\mathscr{P}_{0}(G)$ is a heritable property. By Lemma 4.1, the connected diagrams with the property are precisely those which correspond to free subgroups. Since $\mathcal{G}(i, 1)$ has $G(i)$ vertices, a diagram $\mathscr{D}$ with property $\mathscr{P}_{0}(G)$ must have $|V(\mathscr{D})|$, a multiple of $|G(i)|$. 
Definition. Let $m(G)=1 . c . m .\{|G(i)|: i=1, \ldots, s\}$.

Then $E\left(\mathscr{P}_{0}(G), n\right)$ is zero unless $m(G)$ divides $n$. The calculation of $E\left(\mathscr{P}_{0}(G), m(G) n\right)$ involves only the $C$-type $(m(G) n /|C|, 0, \ldots, 0)$ and the $i$-type $(m(G) n /|G(i)|, 0, \ldots, 0), i=1, \ldots, s$. We get

$$
\begin{aligned}
& E\left(\mathscr{P}_{0}(G), m(G) n\right) /(m(G) n) ! \\
& \quad=\left\{(m(G) n /|C|) !|C|^{m(G) n /|C|}\right\}^{s-1} / \prod_{i=1}^{s}\left\{(m(G) n /|G(i)|) !|G(i)|^{m(G) n /|G(i)|}\right\} .
\end{aligned}
$$

We observe that the right-hand side depends only on the orders $|C|$ and the $|G(i)|$ of the groups appearing in (1) and not on their structures. Indeed, most of the factors depend on ratios of these, e.g. $m(G) /|C|$. We write $G^{*}$ for the free product of cyclic groups of orders $|G(i)| /|C|, i=1, \ldots, s$. Then $m\left(G^{*}\right)$ is defined (since $G^{*}$ is a free product) and $m\left(G^{*}\right)=m(G) /|C|$. Further,

$$
\begin{aligned}
E\left(\mathscr{P}_{0}(G),\right. & m(G) n) /(m(G) n) ! \\
& =|C|^{m\left(G^{*}\right) \mu\left(G^{*}\right) n} E\left(\mathscr{P}_{0}\left(G^{*}\right), m\left(G^{*}\right) n\right) /\left(m\left(G^{*}\right) n\right) !,
\end{aligned}
$$

where

$$
\mu\left(G^{*}\right)=s-1-\sum_{i=1}^{s}|C| /|G(i)|=-1+\sum_{i=1}^{s}\left\{1-(|G(i)| /|C|)^{-1}\right\} .
$$

Since $s>2$, and, for each $i,|G(i)| /|C|>2, \mu\left(G^{*}\right)>0$ except when $G^{*}=Z_{2} * Z_{2}$ (when $\mu\left(G^{*}\right)=0$ ). Except in the last case, $\mu\left(G^{*}\right)$ is a constant multiple of the hyperbolic area of the Fuchsian group isomorphic to $G^{*}$.

Using (10), and Proposition 1.3 applied to $G$ and to $G^{*}$, we have

TheOREM 4.2. For the group $G$ defined by $(1), N_{0}(G, n)$ is zero unless $m(G)$ divides $n$ and

$$
N_{0}(G, m(G) n)=|C|^{1+m\left(G^{*}\right) \mu\left(G^{*}\right) n} N_{0}\left(G^{*}, m\left(G^{*}\right) n\right) .
$$

There is an algebraic proof of Theorem 4.2 when $C$ is normal and $G^{*}$ is the free product of the groups $G(i) / C$ (note that, as above, the value of the right-hand side of (10) depends on the orders of the factors but not upon their structures). The general case of Theorem 4.2 seems to depend on the diagram argument.

We have chosen $G^{*}$ as a free product of cyclic groups so that the results of [5] apply. From (5) of [5] and Theorem 4.2 above, we get

TheOrem 4.3. Let $G$ be the group defined by (1). Then

(i) if $\mu\left(G^{*}\right)>0$,

$$
\begin{aligned}
N_{0}(G, m(G) n)(|C| / 2 \pi)\left\{|C| / \mu\left(G^{*}\right)\right\}^{m\left(G^{*}\right) \mu\left(G^{*}\right) n} \\
\cdot\left\{\left(\mu\left(G^{*}\right)^{-1} \prod_{i=1}^{s}|G(i)| /|C|\right)\right\}^{1 / 2}\left(m\left(G^{*}\right) \mu\left(G^{*}\right) n\right) !
\end{aligned}
$$

(ii) if $\mu\left(G^{*}\right)=0$, i.e. $G^{*}=Z_{2} * Z_{2}$,

$$
N_{0}(G, m(G) n)=|C| \text {. }
$$

We note that there are recurrence relations for the $N_{0}(G, n)$ derived from Theorem 4.2 and the results of [5]. These do not involve the $E\left(\mathscr{P}_{0}(G), n\right)$. 
5. The Case $C=\{1\}$. We note that the definition of $G$ and the above calculations do not require that $C$ be nontrivial. Thus, our results apply to the case where $G$ is a free product of finite groups. In this case, $C=\{1\}$, so that there is only one connected $C$-diagram (a single vertex). This produces some simplification in the formula for $N(G, n)$-the sum over $k$ disappears $(m(0)=1, r(1)=n, c(1)=1)$ and the condition on $i$-types is simply that $n=\sum g(i, j) w(i, j)$. If, further, each $G(i)$ is cyclic, then more simplification occurs since the $g(i, j)$ are precisely the divisors of $|G(i)|$ occurring once each. This produces the formulae of [4].

Department of Mathematics

University Gardens

University of Glasgow

Glasgow G12 8QW, Scotland

1. H. S. M. COXeter \& W. O. J. MOSER, Generators and Relations for Discrete Groups, SpringerVerlag, Berlin, 1965. MR 30 \#4818.

2. W. IMRICH, "Subgroup theorems and graphs," Combinatorial Mathematics V, Lecture Notes in Math., Vol. 622, Springer-Verlag, Berlin and New York, 1977, pp. 1-27. MR 57 \#2980.

3. W. IMrich, "On the number of subgroups of given index in $S L_{2}(Z)$," Arch. Math., v. 31, 1978, pp. 224-231.

4. M. Newman, "Asymptotic formulas related to free products of cyclic groups," Math. Comp., v. 30, 1976, pp. 838-846. MR 57 \#5930.

5. W. W. Stothers, "Free subgroups of the free product of cyclic groups," Math. Comp., v. 32, 1978, pp. 1274-1280.

6. K. Wohtrarrt, “Über einen Satz von Dey und die Modulgruppe," Arch. Math., v. 29, 1977, pp. 455-457. 